\title{
Time-resolved UVES observations of a stellar flare on the planet host HD 189733 during primary transit ${ }^{\star}$
}

\author{
T. Klocová ${ }^{1,2}$, S. Czesla ${ }^{1}$, S. Khalafinejad ${ }^{1}$, U. Wolter $^{1}$, and J. H. M. M. Schmitt ${ }^{1}$ \\ 1 Hamburger Sternwarte, Universität Hamburg, Gojenbergsweg 112, 21029 Hamburg, Germany \\ 2 Astronomical Institute ASCR, Fričova 298, 25165 Ondř̌ejov, Czech Republic \\ e-mail: klocova@asu.cas.cz
}

Received 15 November 2016 / Accepted 5 July 2017

\section{ABSTRACT}

\begin{abstract}
Context. HD 189733 is an exoplanetary system consisting of a transiting hot Jupiter and an active K2V-type main sequence star. Rich manifestations of a stellar activity, like photometric spots or chromospheric flares were repeatedly observed in this system in optical, UV and X-rays.

Aims. We aim to use VLT/UVES high resolution $(R=60000)$ echelle spectra to study a stellar flare.

Methods. We have performed simultaneous analyses of the temporal evolution in several chromospheric stellar lines, namely, the Ca II H \& K lines (3933, $3968 \AA), \mathrm{H} \alpha(6563 \AA), \mathrm{H} \beta(4861 \AA$ ) $, \mathrm{H} \gamma(4341 \AA), \mathrm{H} \delta(4102 \AA), \mathrm{H} \epsilon(3970 \AA)$, the Ca II infrared triplet lines (8498, 8542 and $8662 \AA)$, and He I D3 (5875.6 $)$ ). Observations were carried out with a time resolution of approximately 1 min for a duration of four hours, including a complete planetary transit.

Results. We determine the energy released during the flare in all studied chromospheric lines combined to be about $8.7 \times 10^{31} \mathrm{erg}$, which puts this event at the upper end of flare energies observed on the Sun. Our analysis does not reveal any significant delay of the flare peak observed in the Balmer and Ca II H \& K lines, although we find a clear difference in the temporal evolution of these lines. The He I D3 shows additional absorption possibly related to the flare event. Based on the flux released in Ca II H \& K lines during the flare, we estimate the soft X-ray flux emission to be $7 \times 10^{30} \mathrm{erg}$.

Conclusions. The observed flare can be ranked as a moderate flare on a K-type star and confirms a rather high activity level of HD 189733 host star. The cores of the studied chromospheric lines demonstrate the same behavior and let us study the flare evolution. We demonstrate that the activity of an exoplanet host star can play an important role in the detection of exoplanet atmospheres, since these are frequently discovered as an additional absorption in the line cores. A possible star-planet interaction responsible for a flare occurrence during a transit can neither be confirmed nor ruled out.
\end{abstract}

Key words. stars: chromospheres - stars: flare - stars: activity - techniques: spectroscopic - planetary systems

\section{Introduction}

Because of its proximity to Earth $(d=19.3 \mathrm{pc})$ and apparent brightness $(V=7.7 \mathrm{mag})$, HD 189733 has become one of the most studied exoplanetary systems (e.g., Madhusudhan et al. 2014, and references therein). Its host star, HD $189733 \mathrm{~A}$, is a cool star of spectral type $\mathrm{K} 2 \mathrm{~V}$, which shows the usual signatures of magnetic activity. Photometric observation made with HST by Pont et al. (2007) show flux residuals during a transit, which can be assigned to a spot complex on the stellar surface (with the size in longitude of at least $80000 \mathrm{~km}$ ) that is occulted by the transiting planet. An extensive spectroscopic study of HD 189733 by Boisse et al. (2009) shows activity in several of the "classic" activity indicators such as $\mathrm{Ca}$ II $\mathrm{H} \& \mathrm{~K}, \mathrm{H} \alpha$, and He I D3. With their time series spanning more than 300 days Boisse et al. (2009) also detect a modulation in the chromospheric and photospheric (spotted surface) activity tracers with a 12 day period, identified as the rotation period of the star; this period had previously been estimated by Henry \& Winn (2008) as $P_{\text {rot }}=11.953 \pm 0.009$ days, based on an extensive long-term observing campaign covering 18 complete cycles over approximately 400 days.

* Based on observations made with ESO Telescopes at the La Silla Paranal Observatory under programme ID 089.D-0701(A).
The first discovery of transits of a hot Jupiter planet orbiting around HD $189733 \mathrm{~A}$ is reported by Bouchy et al. (2005), and a little later Bakos et al. (2006) report the discovery of an additional M-type stellar companion HD 189733 B in the system in a 216 AU orbit. The basic characteristics of the star and the planet are summarized in Table 1. Currently HD $189733 \mathrm{~A}$ is one of the most active exoplanetary host stars known. Gray et al. (2003) measure a strength of the chromospheric activity (described in more detail in Sect. 3.2) of $\log R_{\mathrm{HK}}^{\prime}=-4.553$ and Knutson et al. (2010) find a similar value of $\log R_{\mathrm{HK}}^{\prime}=-4.501$. These values classify HD 189733 as an active star, according to the classification suggested by Henry et al. (1996).

X-ray emission from HD $189733 \mathrm{~A}$ has been detected with the EXOSAT and ROSAT satellites (at a time when HD $189733 \mathrm{~A}$ was not known as a planet host), and Cutispoto et al. (2000) quote typical X-ray luminosity levels in the range $1.5-2.5 \times 10^{28} \mathrm{erg} / \mathrm{s}$ from these observations. Comparing this value to the X-ray luminosity distribution function observed for $\mathrm{G}$ and $\mathrm{K}$ dwarfs in the solar neighborhood (Schmitt et al. 1995; Schmitt 1997) shows that - not surprisingly - HD $189733 \mathrm{~A}$ is located in the high-luminosity part of the $\mathrm{X}$-ray luminosity distribution function and thus HD $189733 \mathrm{~A}$ is a very active star also from the X-ray point of view.

The existence of a massive exoplanet on a close-in orbit around an active star makes HD 189733 a very promising target 
Table 1. Top part: properties of the host star HD 189733 A. Bottom part: properties of the transiting planet HD $189733 \mathrm{~B}$.

\begin{tabular}{|c|c|c|}
\hline Property & Value & Source $^{a}$ \\
\hline$T_{\text {eff }}[\mathrm{K}]$ & $5040 \pm 50$ & $\mathrm{~T}$ \\
\hline $\log \left(g\left[\mathrm{~cm} \mathrm{~s}^{-2}\right]\right)$ & $4.587_{-0.015}^{+0.014}$ & $\mathrm{~T}$ \\
\hline$[\mathrm{Fe} / \mathrm{H}]$ & $-0.04^{-0.013} \pm 0.08$ & $\mathrm{~B} / \mathrm{T}$ \\
\hline$v \sin (i)\left[\mathrm{km} \mathrm{s}^{-1}\right]$ & $3.5 \pm 1$ & B \\
\hline$M_{\mathrm{S}}\left[M_{\odot}\right]$ & $0.806 \pm 0.048$ & $\mathrm{~T}$ \\
\hline$R_{\mathrm{S}}\left[R_{\odot}\right]$ & $0.756 \pm 0.018$ & $\mathrm{~T}$ \\
\hline Age [Gyr] & 0.6 & M \\
\hline Distance [pc] & $19.5 \pm 0.3$ & $\mathrm{H}$ \\
\hline$P_{\text {orb }}[$ days $]$ & $2.21857567 \pm 1.5 \times 10^{-7}$ & A \\
\hline$M_{\mathrm{p}}\left[M_{\mathrm{J}}\right]$ & $1.144_{-0.056}^{+0.057}$ & $\mathrm{~T}$ \\
\hline$R_{\mathrm{p}}\left[R_{\mathrm{J}}\right]$ & $1.138 \pm 0.027$ & $\mathrm{~T}$ \\
\hline$T_{0} \mathrm{MJD}_{\mathrm{HJD}, \mathrm{UTC}}$ & 56109.26164 & This work \\
\hline$a[\mathrm{AU}]$ & $0.03099_{-0.00063}^{+0.0006}$ & $\mathrm{~T}$ \\
\hline$i[\mathrm{deg}]$ & $85.58 \pm 0.06$ & $\mathrm{~T}$ \\
\hline
\end{tabular}

References. (a) B: Bouchy et al. (2005), T: Torres et al. (2008), A: Agol et al. (2010), M: Melo et al. (2006), H: Hipparcos, van Leeuwen (2007).

for the study of star-planet interactions. The possible influence on the chromospheric and coronal activity caused by the presence of a close-in Jupiter-like planet is still a matter of ongoing studies and debates. For example, Pillitteri et al. (2010) present a study of an X-ray flare event observed with XMM-Newton, occurring $3 \mathrm{ks}$ after the end of the planetary secondary transit (at orbital phase $\sim 0.54$ ); their analysis suggests flare temperatures of $\sim 10 \mathrm{MK}$, similar to temperatures of solar flares, yet the size of the flaring loop is estimated to be on the order of the stellar radius. Using again XMM-Newton, the same group of authors report a further flare observed near secondary transit (at phase 0.52; Pillitteri et al. 2011) and Pillitteri et al. (2014) report the occurrence of a third flare at phase 0.65 , with similar characteristics as the previous two flares, with a loop of length of four stellar radii at the location of the flare.

Using HST/STIS, Lecavelier des Etangs et al. (2012) find significant variations in an evaporating atmosphere of HD 189733 imprinted on the Ly $\alpha$ line of HD 189733 and suggest that these changes could have been caused by an increased flow of charged particles released during an X-ray flare detected by the Swift satellite $9 \mathrm{~h}$ before the HST observation. Recently Pillitteri et al. (2015) presented new FUV observation with HST/COS of HD 189733, observing HD 189733 during five consecutive HST orbits, covering the orbital phase of $0.5-0.63$ and detecting signatures of enhanced activity in the form of variability in the lines from the elements $\mathrm{Si}, \mathrm{C}, \mathrm{N}$, and $\mathrm{O}$.

In this paper we present ground-based observations of HD 189733 using VLT/UVES and covering a primary transit. Directly in the middle of the transit, a flare occurred, that was followed with a high temporal cadence thus allowing a detailed analysis of this particular stellar flare. Since the flare itself causes changes several times bigger in the line cores than the exoplanetary transit, in this paper we focus exclusively on the flare analysis. Flares are thought to be caused by a sudden energy release in the stellar corona and observed as a brightening across the whole electromagnetic spectrum (for review see e.g., Benz 2008). Their occurrence is unpredictable in nature and their observation requires either long term monitoring or some kind of luck (or bad luck, especially if one intends to use the data for a different purpose). The energy released in solar flares is generally between $10^{29}-3 \times 10^{32} \mathrm{erg}$, but also more energetic events seem to have been observed in several cases (e.g. reported by Notsu et al. 2013). The energies of stellar flares can exceed these solar values by orders of magnitude. The observed stellar chromospheric activity related to a flare arises essentially from the transfer of energy from the corona to the chromosphere, which occurs via accelerated particles (electrons) traveling along magnetic field lines. The subsequent heating of the chromosphere leads to an increasing level of excitation of $\mathrm{H} \alpha$ and other chromospheric lines.

In our analysis, we focus on the temporal evolution of chromospheric lines visible in the optical and the determination of energy released via these lines. In Sect. 2 we describe the observations and data reduction. In Sect. 3 we describe the analysis of the data, the behavior of the chromospheric lines, and their energy budget. In Sect. 4 we discuss our results and in Sect. 5 we give the conclusions.

\section{Observations and data reduction}

Here, we briefly summarize the most relevant aspects of the data processing; a more detailed description of the observations and data reduction can be found in Czesla et al. (2015). The spectroscopic echelle observations of HD 189733 were obtained during the night of July 1/2, 2012, with the UVES spectrograph mounted at the VLT-UT2 telescope in Chile. The data set consists of 244 individual spectra, covering four hours of observation. The exposure time for the first 29 spectra was $30 \mathrm{~s}$ and the rest was exposed for $45 \mathrm{~s}$, resulting in a temporal resolution of approximately $1 \mathrm{~min}$. The observations cover an entire primary transit of HD $189733 \mathrm{~B}(\sim 110 \mathrm{~min})$ plus some pre- and post-transit time. For the data reduction, the UVES pipeline in version 5.2.0 was used. We applied the standard reduction procedure, and also flux calibrated the spectra using the master response files.

Figure 1 shows the temporal evolution of airmass, seeing, the observed flux on the blue chip, and the spectral resolution; the duration of the transit is also indicated. During the first half of the observations, the seeing varies between $0.5^{\prime \prime}$ and $0.6^{\prime \prime}$ and decreases afterwards. The slit width was $1^{\prime \prime}$ for the blue and $0.7^{\prime \prime}$ for the red arm, still larger but on the same order as the seeing disk. We studied the temporal changes in the line profiles using telluric spectral lines. In particular, we selected 15 water lines in the range from 6288 to $7308 \AA$ and derive the median instrumental resolution by measuring their width. The spectral resolution varied during the observation as a result of the changing slit illumination when the seeing disk moves in the slit (see Fig. 1 and Czesla et al. 2015).

Czesla et al. (2015) compare the integrated flux from the individual chips with model spectra (Castelli \& Kurucz 2004; Kurucz 1970) and a blackbody spectrum and find that they generally agree well (see their Table 4); they further attribute the remaining variations in the observed flux of about $20 \%$ (also shown in Fig. 1) to slit losses and the residual influence of airmass and atmospheric conditions. Clearly, the residual flux evolution defines a lower error limit for any flux measurement based on these spectra.

Figure 1 shows a visible evolution in the width of the telluric lines during the observing night, related to changing observing conditions. Since variability in the telluric lines can affect the results of a spectroscopic line analysis, and, in particular, also affects the regions around the activity sensitive $\mathrm{H} \alpha$ and Ca II IRT 


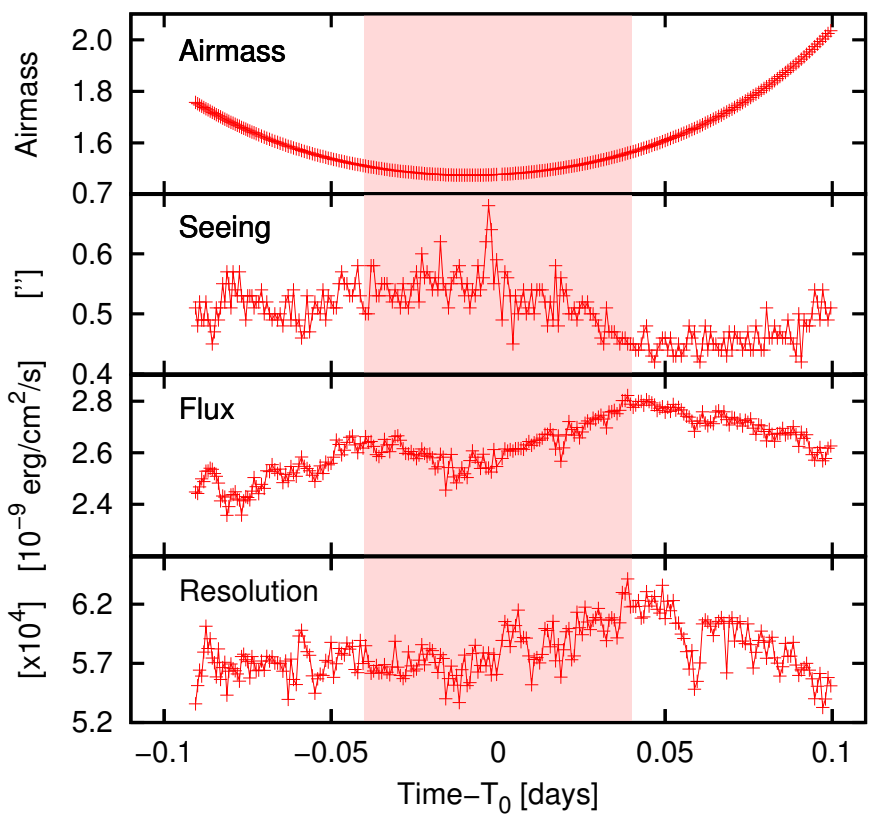

Fig. 1. Observing conditions and evolution of spectral resolution. From top to bottom: temporal evolution of the airmass, seeing, spectral flux measured on a blue chip, and instrumental resolution. Red crosses represent single data points. The shaded area indicates the transit duration and the transit mid-time, $T_{0}$, refers to $\mathrm{MJD}_{\mathrm{HJD}, \mathrm{UTC}}=56109.26164$. This value is used in all the following figures.

triplet lines, a correction of the science spectra for telluric lines is necessary. To remove these lines we used the Molecfit code ${ }^{1}$ (Kausch et al. 2015; Smette et al. 2015). Molecfit works by fitting synthetic telluric transmission spectra to user-specified sections of the astronomical data. Based on the thus derived atmospheric conditions, a synthetic transmission spectrum is then calculated for the entire spectral range. As fitting ranges we selected several telluric molecular bands occurring in the observed spectrum and fit for the three molecules $\mathrm{H}_{2} \mathrm{O}, \mathrm{O}_{2}$ and $\mathrm{O}_{3}$, since these are the most relevant telluric contributors in the optical range. The resulting model transmission spectrum is then used to correct the pipeline-reduced spectrum. The residuals in the spectrum after applying the Molecfit correction are within 5\% for the strongest water lines.

\section{Analysis of chromospheric lines}

The cores of strong spectral lines are often formed in the chromosphere and can thus be used to study the processes occurring in this atmospheric layer. Our UVES spectra cover a wide wavelength range (3760-4980, 5700-7530 , and 7650-9460 ) with a spectral resolution of 60000 . Therefore, the chromosphere of HD 189733 can be studied in many chromospheric lines, formed at different atmospheric heights. In our analysis, we focused on the Ca II H \& K lines $(3933,3968 \AA$ ), the Ca II IR triplet lines $(8498,8542,8662 \AA)$, the Balmer series with $\mathrm{H} \alpha(6563 \AA), \mathrm{H} \beta(4861 \AA), \mathrm{H} \gamma(4341 \AA), \mathrm{H} \delta(4102 \AA)$, and $\mathrm{H} \epsilon(3970 \AA)$, and the He I D3 line (5876 $)$.

\footnotetext{
1 http://www. eso.org/sci/software/pipelines/skytools/ molecfit
}

\subsection{Chromospheric activity indicators}

The Ca II H \& K resonance lines are among the most pronounced spectral features in spectra of late type stars. They result from transitions from $4{ }^{2} \mathrm{~S}_{1 / 2}$ to $4{ }^{2} \mathrm{P}_{1 / 2}^{\mathrm{o}}$ and $4{ }^{2} \mathrm{~S}_{1 / 2}$ to $4{ }^{2} \mathrm{P}_{3 / 2}^{\mathrm{o}}$, respectively and have been regularly used in chromospheric studies, such as the long-term, ground-based survey of stellar activity initiated by O.C. Wilson to analyze the temporal evolution of activity levels and detect analogs of the Sun's 11 year activity cycle in other stars (e.g., Wilson 1968; Baliunas et al. 1995). Several indices such as the Mount-Wilson S-index have been introduced to quantitatively describe the $\mathrm{Ca}$ II $\mathrm{H} \& \mathrm{~K}$ line emission. In our analysis, we focus on the $\log R_{\mathrm{HK}}^{\prime}$ index (Noyes et al. 1984), described in more detail in Sect. 3.2.

The same ion gives rise to the Ca II IR triplet lines, which are strong spectral features in the near infrared region in the spectra of late type stars. They are formed in the lower levels of the chromosphere and arise from the transitions $4^{2} \mathrm{P}_{3 / 2}^{\mathrm{o}}$ to $3^{2} \mathrm{D}_{3 / 2}$ at $8498 \AA, 4^{2} \mathrm{P}_{3 / 2}^{\mathrm{o}}$ to $3^{2} \mathrm{D}_{5 / 2}$ at $8542 \AA$, and $4^{2} \mathrm{P}_{1 / 2}^{\mathrm{o}}$ to $3^{2} \mathrm{D}_{3 / 2}$ at $8662 \AA$. The Ca II IRT lines were recognized and used as activity indicators for example, by Linsky et al. (1979) and Chmielewski (2000).

The cores of the Balmer series lines, most importantly, the $\mathrm{H} \alpha$ line, are also commonly used activity indicators. The $\mathrm{H} \alpha$ line has regularly been used in ground-based studies of solar flares (e.g., Canfield et al. 1984; Leka et al. 1993; Lang 2009) and flares observed on other stars (e.g., Fuhrmeister et al. 2008; Lalitha et al. 2013). In the Sun it is formed in the middle of the chromosphere (Vernazza et al. 1981).

The He I D3 line, which is actually a multiplet resulting from the transitions between the $2^{3} \mathrm{P}^{\mathrm{o}}$ and the $3^{3} \mathrm{D}$ terms, is extremely weak in the quiescent solar photosphere, where it cannot be formed because of its high excitation potential of about $21 \mathrm{eV}$ (e.g., Landman 1981). On the Sun, the He I D3 line appears in absorption in eruptive filaments, flare ejecta, and weak flares and turns into emission only in intense flares (Feldman et al. 1983; Zirin 1988, Sect. 11.3). It shows strong spatial correlation with solar plage regions (Landman 1981). While the details of its formation are still debated (e.g., Athay \& Johnson 1960; Zirin 1975; Andretta et al. 1995; Lanzafame \& Byrne 1995), its absence in the quiet photospheres of the Sun and other late-type stars makes it an excellent indicator of magnetic activity.

The relevant He levels can be populated by photoionzation followed by recombination (P-R mechanism) or collisional excitation in sufficiently hot and dense plasma (e.g., Andretta et al. 1995; Lanzafame \& Byrne 1995). To be observable in emission in solar flares, the line requires a temperature of $>15000 \mathrm{~K}$ and a density in excess of $10^{14} \mathrm{~cm}^{-3}$ (Feldman et al. 1983).

\subsection{Temporal evolution of the chromospheric activity index $\log R_{H K}^{\prime}$}

To study the temporal evolution and energetics of the $\mathrm{Ca}$ II $\mathrm{H} \& \mathrm{~K}$ lines, we determine the chromospheric index $\log R_{\mathrm{HK}}^{\prime}$ for the entire sequence of observations. The $R_{\mathrm{HK}}^{\prime}$ index is defined as the ratio of the chromospheric surface flux in the $\mathrm{Ca}$ II $\mathrm{H} \& \mathrm{~K}$ line cores, $F_{\mathrm{HK}}^{\prime}$, and the bolometric flux of the star through

$R_{\mathrm{HK}}^{\prime}=\frac{F_{\mathrm{HK}}^{\prime}}{\sigma T_{\mathrm{eff}}^{4}}$,

where $\sigma$ is the Stefan-Boltzmann constant and $T_{\text {eff }}$ the effective temperature. The $\log R_{\mathrm{HK}}^{\prime}$ index is based on the Mount Wilson 


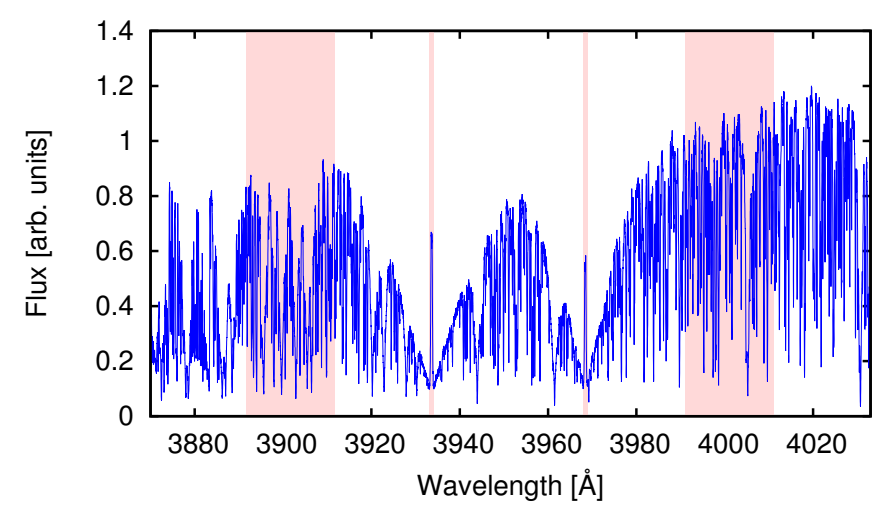

Fig. 2. Spectrum around the Ca II H \& K lines. Shaded areas represent the two reference bands and the two $1 \AA$ wide intervals centered on the $\mathrm{Ca}$ II H \& $\mathrm{K}$ line cores used for the determination of the $\log R_{\mathrm{HK}}^{\prime}$ index.

S-index $\left(S_{\mathrm{HK}}\right)$, from which it mainly differs by excluding photospheric emission and by its normalization (for $S_{\mathrm{HK}}$, see e.g., Middelkoop 1982; Wright et al. 2004).

To derive the $\log R_{\mathrm{HK}}^{\prime}$ index, we first determined $S_{\mathrm{HK}}$ following the calibration procedure described by Melo et al. (2006). In particular, we measured the fluxes in two $1 \AA$ wide intervals centered on the $\mathrm{H}$ and $\mathrm{K}$ line cores, $F_{\mathrm{H}}$ and $F_{\mathrm{K}}$, and determined the fluxes in two reference bands $C_{\mathrm{H}}(3891.67-3911.67 \AA)$ and $C_{\mathrm{K}}$ (3991.067-4011.067 $\AA$ ). The selected passbands are depicted in Fig. 2. Subsequently, we calculated their so-called $S_{\text {US }}$ index defined as $\left(F_{\mathrm{H}}+F_{\mathrm{K}}\right) /\left(C_{\mathrm{H}}+C_{\mathrm{K}}\right)$ and converted it into the Mount Wilson S-index based on the relation (Melo et al. 2006):

$S_{\mathrm{US}}=0.06111 \times S_{\mathrm{HK}}-0.00341$.

Following this, we transformed $S_{\mathrm{HK}}$ into $\log R_{\mathrm{HK}}^{\prime}$ following the procedure described by Noyes et al. (1984) and Wright et al. (2004), applying a value of $B-V=0.93$ taken from Koen et al. (2010).

Figure 3 shows the temporal evolution of the $\log R_{\mathrm{HK}}^{\prime}$ index for our UVES observations, which indicates a relatively quiescent phase during the first half of the observations, where only little variability is seen, followed by an abrupt rise in the middle of the transit, which subsequently turns into a slow decay, continuing beyond the end of our observing run. We dub the rise and subsequent decay the "primary event". The decay phase of the primary event is interrupted by a smaller, secondary and a tertiary event with a shorter rise and decay phase seen $\sim 56 \mathrm{~min}$ and $\sim 95$ min after the start of a primary event, respectively. All three events are characterized by a short rise and a comparably long decay phase, which is typical for stellar and solar flares (e.g., Benz 2008). Therefore, we interpret the observed temporal behavior in terms of three stellar flares. For all three, Fig. 3 allows us to distinguish three flare phases, namely the rise (or impulsive) phase, the peak of the flare, and a gradual decay phase.

A quantitative analysis of the flares requires defining a quiescent level. Given the observed variability pattern, a truly quiescent phase is hard to identify. After visual inspection of Fig. 3, we used the beginning of the observations as the quiescent phase. In particular, we classified the mean of the first 40 spectra as our quiescent reference spectrum. The corresponding quiescent $\log R_{\mathrm{HK}}^{\prime}$ value of -4.525 is represented by a horizontal line in Fig. 3 .

Similarly, the onset of the primary event cannot be uniquely identified. In Fig. 3, we indicate the definition adopted for the beginning of the flare rise phase and the instant of peak flux.

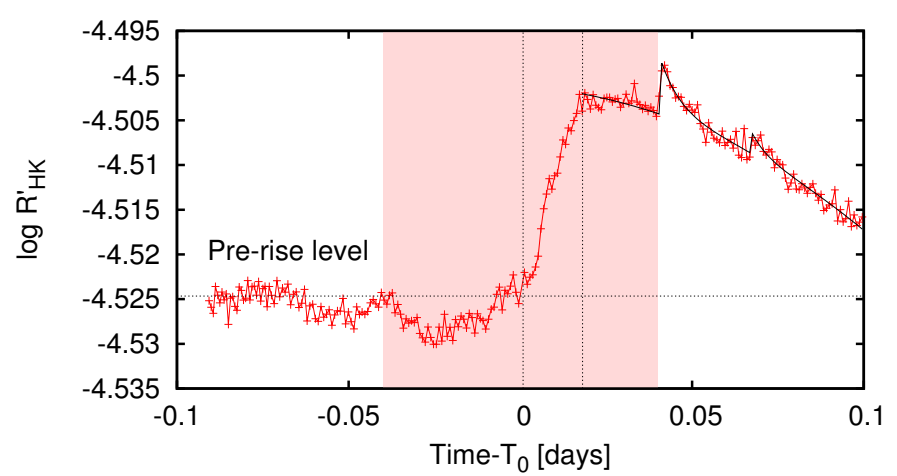

Fig. 3. Temporal evolution of the chromospheric activity index $\log R_{\mathrm{HK}}^{\prime}$. The primary rise and peak phases (dashed, vertical lines), the adopted pre-flare level (horizontal dashed line), and the best fit of the gradual decay phase along with the secondary and tertiary flares are indicated. The center of the $x$ axis (time $=0$ days) corresponds to the middle of the transit $\left(T_{0}[\mathrm{HJD}, \mathrm{UTC}]=2456109.26164\right)$. The shaded pink area indicates the duration of an exoplanetary transit, the $1 \sigma$ error for the data points varies between $3-4 \%$.

These times are determined as an intersection of two linear fits to visually selected sections of the light curve neighboring the flare peak and start. According to our definition, the flare starts 2 min after mid-transit time and shows a 24 min long rise phase.

Along with the data, Fig. 3 shows a fit to the decay part of the light curve, which consists of a third-order polynomial to represent the decay phase of the primary flare. The secondary and tertiary events can be fit using an instantaneous rise phase followed by an exponential decay on top of the polynomial background. Although the decay of the primary flare is not well described by an exponential, it can be characterized by an e-folding time. This quantity is defined as the time after which the flare flux drops to $37 \%$ compared to its maximum. We estimate an e-folding time of approximately $111 \mathrm{~min}$ for the primary event. According to our modeling, the secondary event reaches a peak level of -4.499 in $R_{\mathrm{HK}}^{\prime}$ and shows a decay time of $8.7 \mathrm{~min}$. The tertiary flare shows a slightly shorter e-folding time of $6.5 \mathrm{~min}$. All the derived values are summarized in Table 2.

Figure 3 also shows a dip in the light curve visible at the beginning of the transit and interrupted by the stellar flare in the middle of the transit. This dip can probably be attributed to the center-to-limb variation, which is described in more detail in Czesla et al. (2015).

The rise in $\log R_{\mathrm{HK}}^{\prime}$ associated with the primary flare can directly be observed in the spectrum. In Fig. 4, we show the quiescent reference spectrum of the $\mathrm{Ca}$ II $\mathrm{K}$ line along with a spectrum from the most active flare phase. Clearly, the latter exhibits stronger Ca II H \& K line cores.

\subsection{Time resolved analysis of chromospheric flux residuals}

In this section we present an analysis of the chromospheric core emission observed in the Ca II IRT lines, the lines of the Balmer series, and the He I D3 line, along with an alternative analysis of the emission in the $\mathrm{Ca}$ II $\mathrm{H} \& \mathrm{~K}$ lines based directly on the flux calibrated spectra and not relying on the conversion of chromospheric indices. To study the effect of the flare on chromospherically sensitive lines, we derive the temporal evolution of the flux residuals in their line cores. As detailed in Sect. 3.2, we use the mean of the first 40 spectra as a quiescent reference spectrum to which the remaining spectra are compared to detect residual 


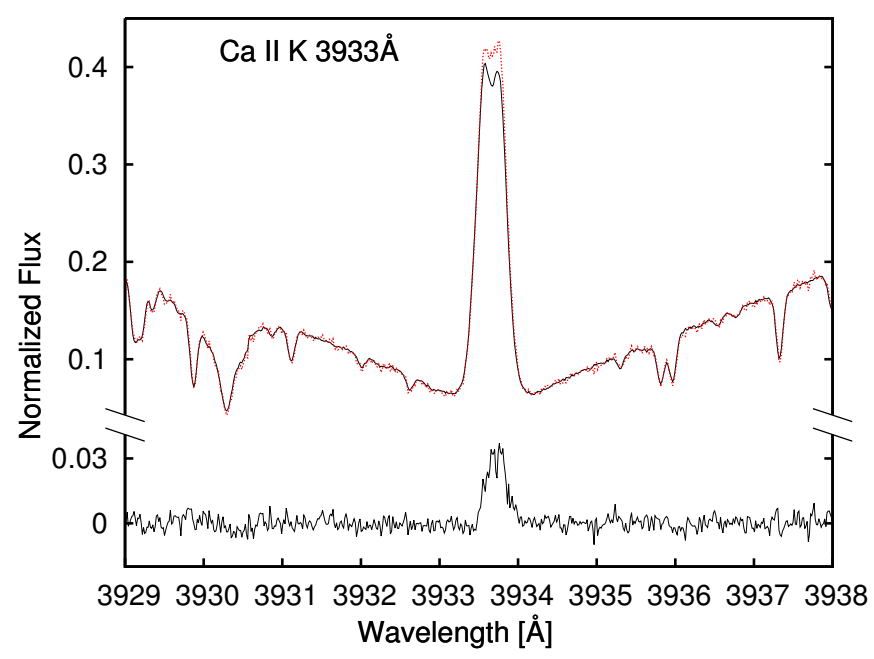

Fig. 4. Activity in the core of the Ca II K line ( $3933 \AA$ ). Upper part: quiescent reference spectrum (solid black line) and spectrum observed during the flare peak (dotted red line). Bottom curve: flux residuals after subtracting the quiescent spectrum from the flare spectrum (solid black line).

Table 2. Characteristics of the flares determined from the $R_{\mathrm{HK}}^{\prime}$ index.

\begin{tabular}{ll}
\hline \hline Parameter & Value \\
\hline$S_{\mathrm{PF}}[\mathrm{MJD}-56109]$ & $0.263 \pm 0.002$ \\
$\Delta t_{\text {rise }}[\mathrm{min}]$ & 24.2 \\
$M_{\mathrm{PF}}[\mathrm{MJD}-56109]$ & $0.2798 \pm 0.0008$ \\
$M_{\mathrm{SF}}[\mathrm{MJD}-56109]$ & $0.3052_{-0.0043}^{+0.0048}$ \\
$M_{\mathrm{TF}}[\mathrm{MJD}-56109]$ & $0.3193_{-0.0093}^{+0.0090}$ \\
$E_{\mathrm{Tot}}\left[10^{31} \mathrm{erg}\right]$ & 1.26 \\
$\tau_{\mathrm{PF}}[\mathrm{min}]$ & 111.14 \\
$\tau_{\mathrm{SF}}[\mathrm{min}]$ & $11.0_{-1.9}^{+1.8}$ \\
$\tau_{\mathrm{TF}}[\mathrm{min}]$ & $7.6_{-1.6}^{+1.9}$ \\
$L_{\mathrm{MP}}\left[10^{27} \mathrm{erg} \mathrm{s}^{-1}\right]$ & 2.06 \\
$L_{\mathrm{MS}}\left[10^{26} \mathrm{erg} \mathrm{s}^{-1}\right]$ & 5.175 \\
$L_{\mathrm{MT}}\left[10^{25} \mathrm{erg} \mathrm{s}^{-1}\right]$ & 3.055 \\
\hline
\end{tabular}

Notes. $S_{\mathrm{PF}}-$ start of the primary flare with $1 \sigma$ confidence limits; $\Delta t_{\text {rise }}-$ duration of the primary rise phase; $M_{\mathrm{PF}}, M_{\mathrm{SF}}, M_{\mathrm{TF}}$ - instant of peak flux of the primary, secondary, and tertiary flare; $E_{\mathrm{Tot}}-$ energy released during a whole event; $\tau_{\mathrm{PF}}, \tau_{\mathrm{SF}}, \tau_{\mathrm{TF}}-$ e-folding times of the primary, secondary, and tertiary flare; $L_{\mathrm{MP}}, L_{\mathrm{MS}}, L_{\mathrm{MT}}$ - peak luminosity of the primary, secondary, and tertiary flare in Ca II $\mathrm{H} \& \mathrm{~K}$. Calculation of $1 \sigma$ errors for $S_{\mathrm{PF}}$ and $M_{\mathrm{PF}}$ are based on Filliben \& McKinney (1972). Errors of $M_{\mathrm{SF}}, M_{\mathrm{TF}}, \tau_{\mathrm{SF}}$ and $\tau_{\mathrm{TF}}$ are determined by MCMC method.

emission similar to the core flux residuals already indicated in Fig. 4.

A comparison of the Ca II IRT lines in our quiescent reference spectrum with synthetic model spectra shown in Fig. 5 clearly demonstrates that the central parts of the lines show a noticeable fill-in also in the quiescent phase. This is comparable to but less pronounced than the cores of the $\mathrm{Ca}$ II $\mathrm{H} \& \mathrm{~K}$ lines and a consequence of the generally high level of stellar activity in HD 189733.

The flare phase is characterized by an increase in the core emission of the chromospheric lines. Figures 4 and 6 show the line cores of the Ca II K line and the shortest-wavelength component of the Ca II IRT ( $8498 \AA$ ) at two different times, namely in the quiescent phase and during the more active phase in the second half of the observation. In the bottom parts of the figures we explicitly show the flux residuals, demonstrating that the core emission is clearly stronger during the later observation for both lines.

To study the evolution of the line cores we subtracted the quiescent reference spectrum from each observed spectrum and thus obtain the residuals in the line cores. Subsequently, we fit these residuals with a Gaussian profile and determine the corresponding excess equivalent width $W_{\text {ex }}$ for all spectral lines under consideration. Since our spectra are flux calibrated, we could directly convert measured excess equivalent width into the flux observed at Earth by multiplying $W_{\text {ex }}$ by the reference continuum flux around the line. Because of the continuum flux evolution in the flux calibrated spectra during the observation run (see Fig. 1), we used an average value for the continuum reference flux based on all 244 spectra.

We carried out this procedure for all the selected lines and the resulting light curves are depicted in Fig. 7. Qualitatively, all but one of the lines show the same behavior as the Ca II H \& $\mathrm{K}$ line cores (cf., Fig. 3), namely, an abrupt rise around the center of the exoplanetary transit followed by a slower decay. In the case of the $\mathrm{Ca}$ II $\mathrm{H} \& \mathrm{~K}, \mathrm{Ca}$ II IRT, $\mathrm{H} \alpha$, and $\mathrm{H} \beta$ lines also the secondary and tertiary event can be identified. Only the He I D3 line shows a somewhat different behavior with hardly any excess emission during the rise phase and a turn into absorption during the decay phase of the primary event; this behavior is discussed in detail in Sect. 3.4.

Based on the core emission light curves shown in Fig. 7, we determine the start of the primary flare and the time of the peak flux for each line individually (we followed the procedure described in Sect. 3.2). The results are indicated in Fig. 7 and the values are summarized in Table 4.

The gradual decay phases of the primary event observed in the Balmer lines can be described by an exponential decay function. In the $\mathrm{H} \gamma, \mathrm{H} \delta$, and $\mathrm{H} \epsilon$ lines no secondary and tertiary event can be identified so that a single exponential is sufficient to model the decay phase. In the case of $\mathrm{H} \alpha$ and $\mathrm{H} \beta$, we modeled the decay phase using a combination of three exponential decay functions representing the primary, secondary, and tertiary events.

In the case of the Ca II lines, the decay of the primary event is not well represented by an exponential. Therefore, we modeled the decay of the primary by a polynomial and superimpose two exponentials representing the secondary and tertiary events. The resulting fits for all lines are depicted in Fig. 7. Based on our modeling, we determine the e-folding times for all events and spectral lines and the peak fluxes for the secondary and tertiary flares. The resulting values are listed in Table 5.

\section{4. $\mathrm{He} I \mathrm{D} 3$}

The excess equivalent width $W_{\mathrm{ex}}$ in the core of the He I D3 line shows a different evolution during the observation than the other studied lines (see Fig. 8, bottom panel). While there may be a small amount of excess emission during the flare rise phase observed in other lines $\left(\sim 0.2 \times 10^{-14} \mathrm{erg} \mathrm{cm}^{-2} \mathrm{~s}^{-1}\right)$, there is no clear flare signature, and the line goes into absorption compared to the pre-flare level during the decay phase of the primary event.

Based on this finding, we checked the behavior of the He I D3 line as a whole by computing its equivalent width, whose evolution is shown in the top panel of Fig. 8. It confirms the diverse behavior of the line. During quiescence a He I D3 EW 

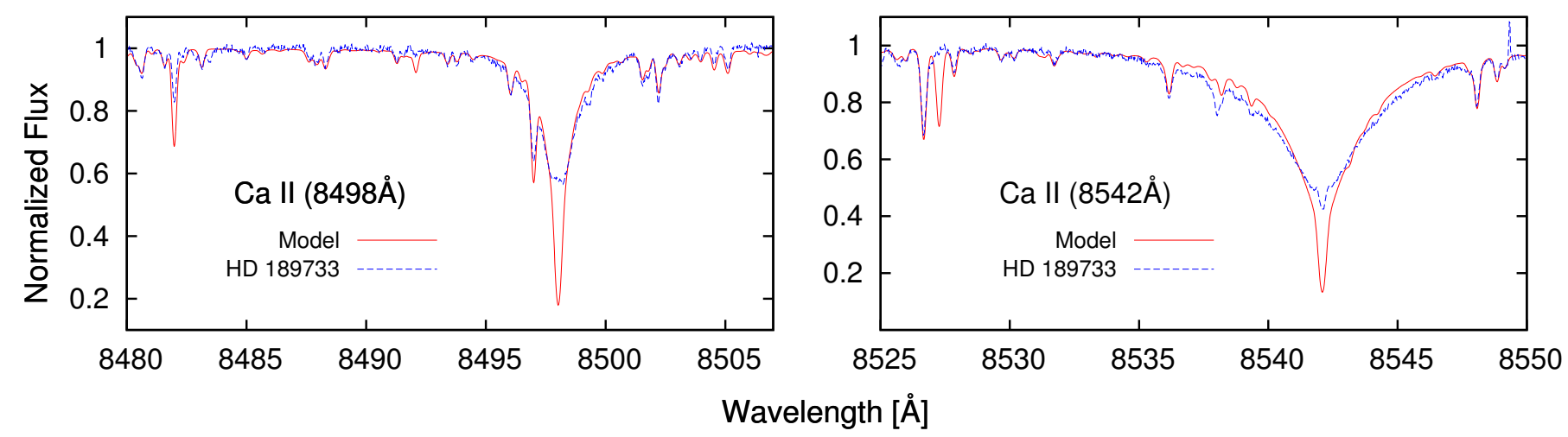

Fig. 5. Comparison of the observed first ( $8498 \AA$ ) and second ( $8542 \AA$ ) Ca II IRT line profiles (blue dashed line) with a photospheric model (red solid line). The model was created using the SPECTRUM program (Gray \& Corbally 1994) based on a Kurucz stellar atmosphere model for $T_{\text {eff }}=5000 \mathrm{~K}, \log g=4.5$, and solar metallicity (Castelli \& Kurucz 2004).

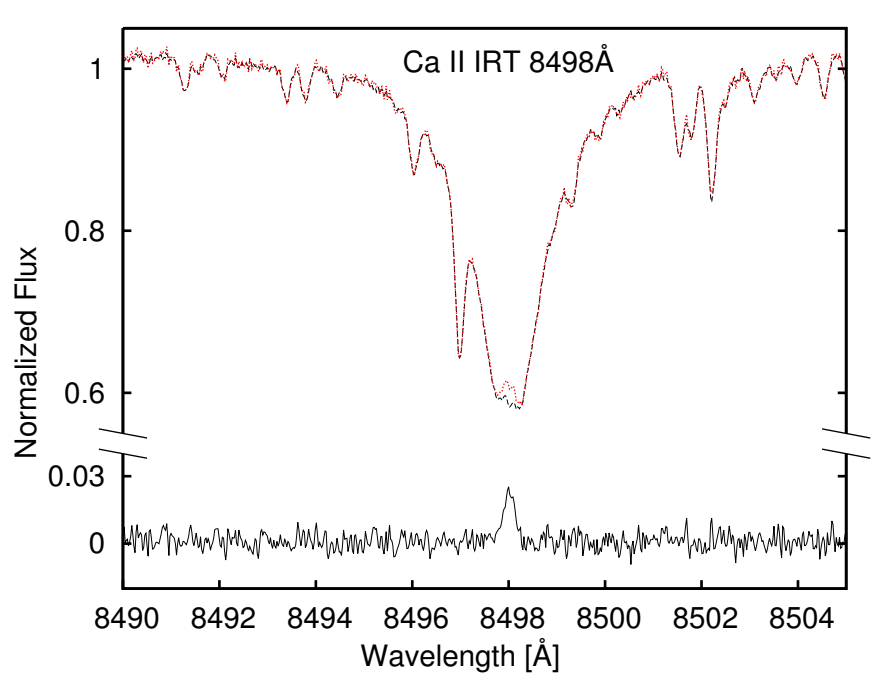

Fig. 6. Activity in the core of the first Ca II IRT line ( $8498 \AA$ A). Upper part: dotted red line - spectrum observed during the activity peak (second half of the observation); dashed black line - quiescent spectrum determined as an average value of 40 spectra obtained at the beginning of the observation. Bottom curve: solid black line in the bottom part: flux residuals after subtracting the quiescent spectrum from the active spectrum.

of $\sim 13.5 \mathrm{~m} \AA$ is measured, corresponding to a surface flux, $F_{\mathrm{D} 3}$, of $51000 \mathrm{erg} \mathrm{cm}^{-2} \mathrm{~s}^{-1}$. These values are likely slightly overestimated because the line is blended (e.g., Saar et al. 1997). The EW remains essentially constant until approximately $35 \mathrm{~min}$ after the mid-transit time and rises afterward. About $70 \mathrm{~min}$ after the mid-transit time the equivalent width seems to stop rising and remains constant until the end of our measurements. The increase in equivalent width is about $1 \mathrm{~m} \AA$. For reference, we reproduced the evolution of the excess equivalent width, $W_{\mathrm{ex}}$, in the core of the He I D3 line in Fig. 8. Both plots show that the absorption in the He I D3 line increases compared to the quiescent level in contrast to the other studied chromospheric lines.

To rule out instrumental effects, we checked neighboring spectral regions and studied the equivalent width of three selected photospheric lines and compare the evolution of continuum regions without strong spectral lines. The resulting light curves are plotted in Fig. 9. They are essentially constant during the observation. None of them shows behavior similar to the
He I D3 line. Therefore, we argue that the observed behavior in the He I D3 line is not instrumental.

\subsection{Chromospheric flare energy budget}

Based on our excess flux measurements, we calculated the energy released during the flare in the individual lines. We converted the measured flux at Earth, $F_{\oplus}$, into luminosity, $L$, using the relation

$L=4 \pi d^{2} \cdot F_{\oplus}$

where $d$ is distance to HD 189733 (see Table 1).

For the primary event, we determined the energy released during the rise phase by integrating the chromospheric excess emission during that phase. Similarly, the energy released during the decay phase was obtained by integrating the measured flux in that phase. As is clear from Figs. 3 and 7, the primary event is not completely covered by our observations. To obtain an estimate of the missing fraction, we extrapolated the evolution of the flare and estimate that about $10 \%$ of the total energy released in the $\mathrm{Ca}$ II $\mathrm{H} \& \mathrm{~K}$ lines remain unobserved. As the estimate of the energy released by the primary event is, however, further complicated by the uncertainty in the definitions of the quiescent level estimation, which is about $2 \%$, the definition of the start of the flare, and the uncertainty in the flux calibration, which is on the order of $20 \%$ (this value is based on the comparison of our flux calibrated spectra with the predicted Kurucz model atmosphere as discussed in Sect. 2 of this paper and in Sect. 5.2 in Czesla et al. 2015), we argue that the missing energy fraction does not significantly influence the resulting overall energy budget and can, therefore, be neglected in the following analysis.

Adding the energy released during the rise and decay phases, we calculated the total energy released during the observed flare event in the individual lines. In the case of the secondary and tertiary flares, the peak fluxes, decay times, and total energies can be directly derived from our modeling of the excess flux by an exponential. The statistical " $1 \sigma$ " error estimate for the energy measurements (for all the lines) is on the order of a few percent from the determined values. Our results are summarized in Tables 3 and 5 .

In the case of the $\mathrm{Ca}$ II $\mathrm{H} \& \mathrm{~K}$ lines, an alternative estimate of the peak flux and released energy can be obtained based on $R_{\mathrm{HK}}^{\prime}$. Combining the effective temperature and stellar radius 

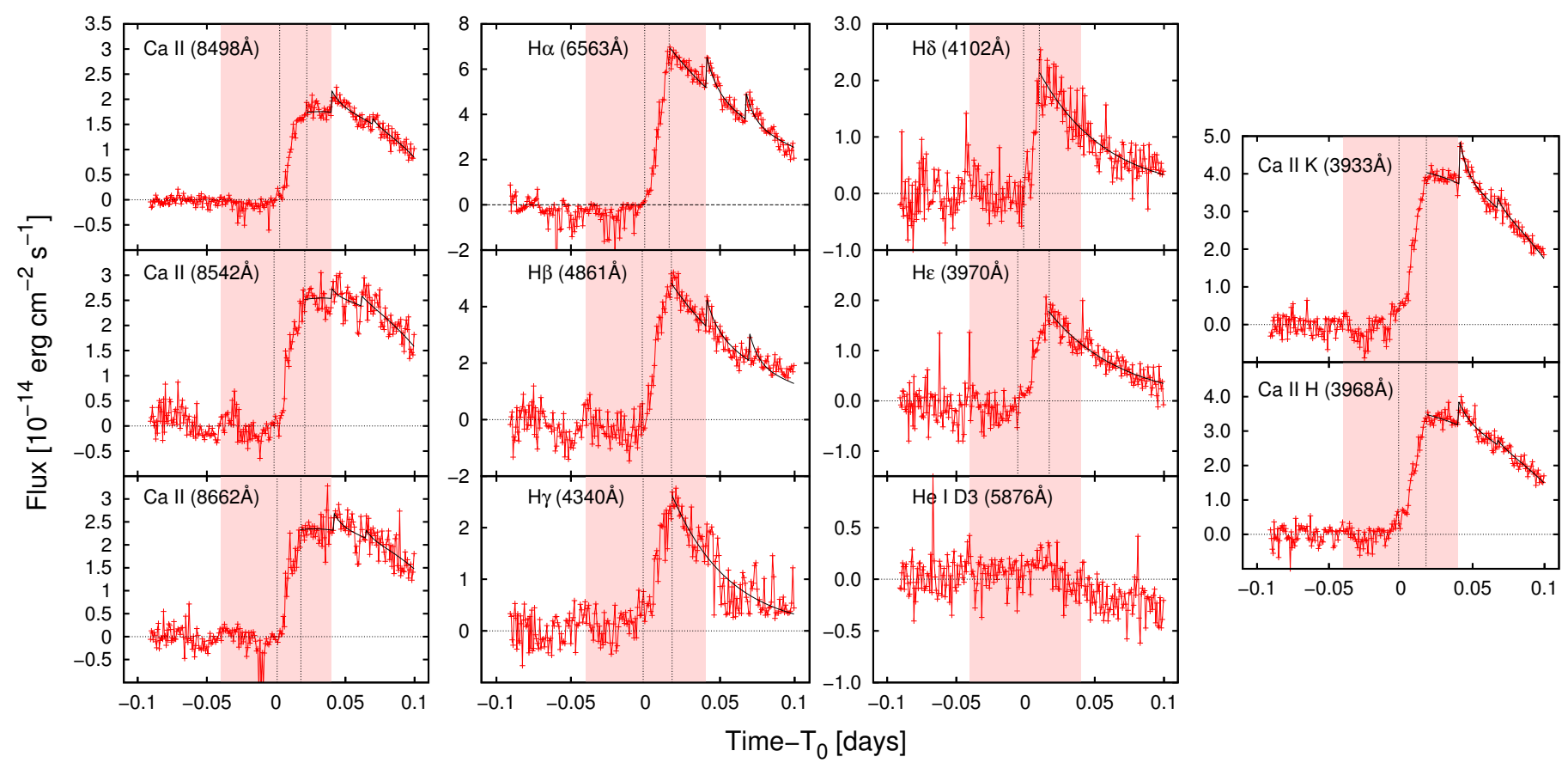

Fig. 7. Evolution of a flare in selected chromospheric optical lines. The $y$ axis is the flux measured at Earth, $x$ axis is time in days. The center of the $x$ axis (time $=0$ days) corresponds to the middle of the transit $\left(T_{0}[\mathrm{HJD}, \mathrm{UTC}]=2456\right.$ 109.26164). Red crosses are the measured flux value. Full black lines represent a best fit of the flare decay phase. Dashed black vertical lines mark a beginning and peak of the flare for each line individually. Horizontal dashed black lines indicate a quiescent zero level. The shaded pink area labels the duration of an exoplanetary transit. The $1 \sigma$ error for the displayed data points is within the $3-6 \%$ range.

Table 3. Energy budget of the flare.

\begin{tabular}{llcccc}
\hline \hline Line & $\begin{array}{l}E_{\text {rise }} \\
{\left[10^{30} \mathrm{erg}\right]}\end{array}$ & $\begin{array}{c}E_{\mathrm{SF}} \\
{\left[10^{27} \mathrm{erg}\right]}\end{array}$ & $\begin{array}{c}E_{\mathrm{TF}} \\
{\left[10^{27} \mathrm{erg}\right]}\end{array}$ & $\begin{array}{c}E_{\mathrm{Tot}} \\
{\left[10^{31} \mathrm{erg}\right]}\end{array}$ & $\begin{array}{c}L_{\mathrm{PF}} \\
{\left[10^{27} \mathrm{erg} \mathrm{s}^{-1}\right]}\end{array}$ \\
\hline $\mathrm{H} \alpha$ & 1.82 & 4.74 & 3.14 & 1.67 & 3.11 \\
$\mathrm{H} \beta$ & 1.81 & 3.60 & 2.74 & 1.56 & 2.18 \\
$\mathrm{H} \gamma$ & 0.91 & - & - & 0.45 & 1.15 \\
$\mathrm{H} \delta$ & 0.26 & - & - & 0.37 & 0.95 \\
$\mathrm{H} \epsilon$ & 0.64 & - & - & 0.35 & 0.79 \\
\hline $\mathrm{Ca}$ II K & 1.33 & 4.21 & 0.89 & 1.17 & 1.80 \\
$\mathrm{Ca}$ II H & 1.17 & 2.81 & 0.55 & 1.00 & 1.55 \\
\hline Ca II IRT $\lambda 8498$ & 0.79 & 2.10 & 0.31 & 0.55 & 0.77 \\
Ca II IRT $\lambda 8542$ & 1.06 & 0.55 & 1.16 & 0.82 & 1.12 \\
Ca II IRT $\lambda 8662$ & 0.71 & 1.22 & 0.51 & 0.75 & 1.04 \\
\hline Total energy & 10.54 & - & - & 8.69 & - \\
\hline
\end{tabular}

Notes. $E_{\text {rise }}$ - energy released during the impulsive phase; $E_{\mathrm{SF}}$ - energy released by the secondary flare; $E_{\mathrm{TF}}$ - energy released by the tertiary flare; $E_{\mathrm{Tot}}$ - energy released during the total flare; $L_{\mathrm{PF}}$ - peak luminosity of the primary flare. $1 \sigma$ error estimation is within $4 \%$ for all the values.

given in Table 1 and the quiescent level of -4.525 for $R_{\mathrm{HK}}^{\prime}$ estimated in Sect. 3.2, we estimated a quiescent chromospheric Ca II H \& K line luminosity of $3.85 \times 10^{28} \mathrm{erg} \mathrm{s}^{-1}$ for HD 189733. The peak flux for the primary event indicated in Fig. 3 yields a value of $2.06 \times 10^{27} \mathrm{erg} \mathrm{s}^{-1}$ for the corresponding peak luminosity. By integrating the energy released by the primary event in excess of the quiescent level, we deduced a value of $1.26 \times 10^{31}$ erg for the total energy released in the Ca II H \& K emission line cores by the primary event. This value is smaller by about $60 \%$ than the overall energy released in $\mathrm{Ca}$ II $\mathrm{H} \& \mathrm{~K}$ lines $E_{\text {Tot }}=2.17 \times 10^{31}$ erg determined with the method described in Sect. 3.3. The two values are nevertheless on the same order and this discrepancy can be attributed to the imperfection in the flux calibration and the derivation of the $R_{\mathrm{HK}}^{\prime}$ index.

\section{Discussion}

The temporal evolution in the cores of the chromospheric lines displayed in Fig. 7 shows a stellar flare, beginning shortly after mid-transit time. While the line evolution is qualitatively similar for all the lines except for the He I D3 line, a more detailed inspection shows quantitative differences between individual lines.

\subsection{Evolution of the Balmer and Ca II lines}

Both the Balmer and Ca II lines show a steep rise phase, followed by a much slower decay phase. The measured durations of the rise phase in all the studied lines are in the range of 20-30 min with a median of $28 \mathrm{~min}$. The peak and subsequent decay clearly 
Table 4. Time characteristics of a flare in studied lines.

\begin{tabular}{lcccc}
\hline \hline Line & $\begin{array}{c}S_{\mathrm{PF}} \\
{[\mathrm{MJD}-56109]}\end{array}$ & $\begin{array}{c}M_{\mathrm{PF}} \\
{[\mathrm{MJD}-56109]}\end{array}$ & $\begin{array}{c}M_{\mathrm{SF}} \\
{[\mathrm{MJD}-56109]}\end{array}$ & $\begin{array}{c}M_{\mathrm{TF}} \\
{[\mathrm{MJD}-56109]}\end{array}$ \\
\hline $\mathrm{H} \alpha$ & $0.2611 \pm 0.0006$ & $0.2775 \pm 0.0004$ & $0.3024_{-0.0004}^{+0.0003}$ & $0.3288_{+0.0004}^{-0.0003}$ \\
$\mathrm{H} \beta$ & $0.2595 \pm 0.0010$ & $0.2790 \pm 0.0006$ & $0.3022_{-0.0001}^{+0.0006}$ & $0.335_{-0.004}^{+0.002}$ \\
$\mathrm{H} \gamma$ & $0.2601 \pm 0.0014$ & $0.2795 \pm 0.0010$ & - & - \\
$\mathrm{H} \delta$ & $0.2602 \pm 0.0010$ & $0.2716 \pm 0.0010$ & - & - \\
$\mathrm{H} \epsilon$ & $0.2560 \pm 0.0014$ & $0.2787 \pm 0.0009$ & - & - \\
\hline $\mathrm{Ca}$ II K & $0.2604 \pm 0.0006$ & $0.2794 \pm 0.0005$ & $0.3086_{-0.0080}^{+0.0297}$ & $0.3217_{-0.0214}^{+0.0230}$ \\
$\mathrm{Ca}$ II H & $0.2601 \pm 0.0007$ & $0.2792 \pm 0.0006$ & $0.3066_{-0.0066}^{+0.0269}$ & $0.3232_{-0.0230}^{+0.0187}$ \\
\hline $\mathrm{Ca}$ II IRT $\lambda 8498$ & $0.2642 \pm 0.0008$ & $0.2838 \pm 0.0010$ & $0.3173_{-0.0172}^{+0.0268}$ & $0.3210_{-0.0204}^{+0.0249}$ \\
$\mathrm{Ca}$ II IRT $\lambda 8542$ & $0.2601 \pm 0.0010$ & $0.2823 \pm 0.0009$ & $0.3046_{-0.0033}^{+0.0053}$ & $0.3235_{-0.0034}^{+0.0053}$ \\
$\mathrm{Ca}$ II IRT $\lambda 8662$ & $0.2624 \pm 0.0011$ & $0.2795 \pm 0.0012$ & $0.3040_{-0.0039}^{+0.0029}$ & $0.3255_{-0.0085}^{+0.0233}$ \\
\hline
\end{tabular}

Notes. $S_{\mathrm{PF}}-$ start of the primary flare with $1 \sigma$ confidence limits; $M_{\mathrm{PF}}-$ peak of the primary flare/the end of the impulsive phase; $M_{\mathrm{SF}}-$ peak of the secondary flare; $M_{\mathrm{TF}}$ - peak of the tertiary flare. Calculation of $1 \sigma$ errors for $S_{\mathrm{PF}}$ and $M_{\mathrm{PF}}$ are based on Filliben \& McKinney (1972). Errors of $M_{\mathrm{SF}}$ and $M_{\mathrm{TF}}$ are determined by MCMC method.

Table 5. Flare duration and e-folding time.

\begin{tabular}{lccccc}
\hline \hline Line & $\begin{array}{c}\Delta t_{\text {rise }} \\
{[\mathrm{min}]}\end{array}$ & $\begin{array}{c}\Delta t_{\mathrm{F}} \\
{[\mathrm{min}]}\end{array}$ & $\begin{array}{c}\tau_{\mathrm{PF}} \\
{[\mathrm{min}]}\end{array}$ & $\begin{array}{c}\tau_{\mathrm{SF}} \\
{[\mathrm{min}]}\end{array}$ & $\begin{array}{c}\tau_{\mathrm{TF}} \\
{[\mathrm{min}]}\end{array}$ \\
\hline $\mathrm{H} \alpha$ & 23.62 & 144.16 & $119.1_{-9.2}^{+3.7}$ & $10.3_{-2.1}^{+1.8}$ & $9.25_{-1.15}^{+0.75}$ \\
$\mathrm{H} \beta$ & 28.08 & 146.47 & $90.8_{-5.5}^{+5.1}$ & $9.36_{-0.29}^{+0.35}$ & $7.56_{-0.43}^{+0.44}$ \\
$\mathrm{H} \gamma$ & 27.94 & 145.60 & $56.5_{-5.0}^{+5.4}$ & - & - \\
$\mathrm{H} \delta$ & 16.42 & 145.44 & $70.2_{-7.2}^{+8.5}$ & - & - \\
$\mathrm{H} \epsilon$ & 32.69 & 151.51 & $72.8_{-9.8}^{+9.5}$ & - & - \\
\hline $\mathrm{Ca}$ II K & 27.36 & 145.17 & 121.43 & $9.7_{-1.3}^{+1.3}$ & $8.2_{-1.2}^{+1.4}$ \\
$\mathrm{Ca}$ II H & 27.50 & 145.60 & 121.53 & $11.0_{-1.9}^{+1.7}$ & $8.0_{-1.0}^{+1.4}$ \\
\hline $\mathrm{Ca}$ II IRT $\lambda 8498$ & 28.22 & 139.70 & 119.88 & $10.9_{-1.9}^{+1.8}$ & $7.6_{-1.6}^{+1.8}$ \\
$\mathrm{Ca}$ II IRT $\lambda 8542$ & 31.97 & 145.60 & 140.22 & $10.8_{-1.8}^{+1.9}$ & $8.7_{-1.8}^{+1.3}$ \\
$\mathrm{Ca}$ II IRT $\lambda 8662$ & 24.62 & 142.29 & 137.72 & $11.1_{-1.7}^{+1.9}$ & $7.9_{-1.9}^{+1.7}$ \\
\hline
\end{tabular}

Notes. $\Delta t_{\text {rise }}$ - duration of an impulsive phase; $\Delta t_{\mathrm{F}}$ - duration of a whole flare; $\tau_{\mathrm{PF}}-$ e-folding time of the primary flare; $\tau_{\mathrm{SF}}-\mathrm{e}$-folding time of the secondary flare; $\tau_{\mathrm{TF}}-\mathrm{e}$-folding time of the tertiary flare. MCMC method was used for the error determination.

differs between the Balmer lines and the Ca II lines. While all Balmer lines show a sharp flare peak, immediately followed by a decay, which is well described by an exponential function, all Ca II lines lack the sharp flare peak, rather they show a "plateau" after the rise phase. Therefore, an accurate definition of the instant of peak flux is difficult in the case of the Ca II lines.

While the $\mathrm{H} \alpha, \mathrm{H} \beta, \mathrm{H} \gamma$, and $\mathrm{H} \epsilon$ lines all peak within about three minutes, the estimated peak of the $\mathrm{H} \delta$ line occurs approximately $10 \mathrm{~min}$ before that (see Table 4). A close visual inspection of the HD 189733 light curve shows, however, that the adopted peak position mainly depends on two data points and the physical peak could also plausibly occur later when the remaining Balmer light curves peak. This appears even more plausible considering that we determined the by far shortest rise phase of $16.4 \mathrm{~min}$ for the $\mathrm{H} \delta$ line. We thus consider the evidence insufficient to claim a significant delay in the flare peak observed in the Balmer lines.

In the Ca II lines, we identified the instant of peak flare flux with the beginning of the plateau phase. According to our definitions, there is a tendency for the $\mathrm{Ca}$ II lines to peak a few minutes after the Balmer lines. For example, the $\mathrm{H} \alpha$ line peaks approximately $2.5 \mathrm{~min}$ before both $\mathrm{Ca}$ II $\mathrm{H} \& \mathrm{~K}$ lines. In an analysis of several flares on AD Leo, Crespo-Chacón et al. (2006) find that the flare peaks in the $\mathrm{Ca}$ II $\mathrm{H} \& \mathrm{~K}$ lines were delayed with respect to that of the Balmer lines by up to $5 \pm 3 \mathrm{~min}$; in particular, this refers to the delay of the Ca II $\mathrm{K}$ line with respect to $\mathrm{H} \beta$. If anything, our observation indicates a similar trend. However, the morphology of the Ca II light curves prevents an accurate identification of the start of the plateau phase. So while the Ca II lines definitely show a different temporal evolution than the Balmer lines, a delay in the instant of peak flux cannot be uniquely detected.

The delay in the range of 5-20 min of the flare peak in $\mathrm{Ca}$ II $\mathrm{H} \& \mathrm{~K}$ lines with respect to Balmer lines is frequently observed in flares on M-type stars (e.g., García-Alvarez et al. 2002; Fuhrmeister et al. 2008; Hawley \& Pettersen 1991), on the other hand, there are also observations of flares, where Balmer lines peak together with the Ca II lines (e.g. Kowalski et al. 2015, solar flare). The evolution of the optical chromospheric lines during a flare show an apparently diverse behavior and is a subject 


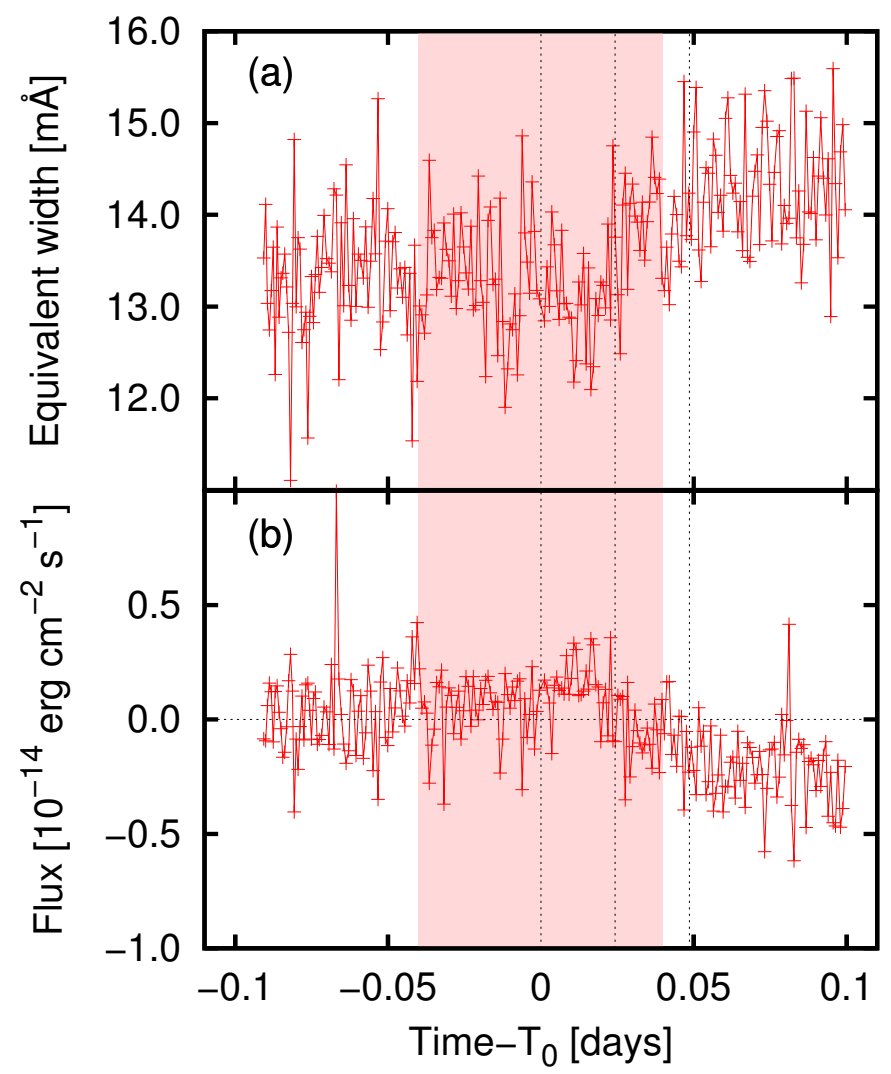

Fig. 8. Panel $a$ : evolution of the He I D3 line equivalent width. Panel $b$ : evolution of the excess equivalent width, $W_{\mathrm{ex}}$, in the core of the He I D3 line converted into the flux measured at Earth (see also Fig. 7). Shaded pink area indicates the duration of an exoplanetary transit. Meaning of dashed lines from left to right: middle of the transit $T_{0}[\mathrm{HJD}, \mathrm{UTC}]=$ 2456 109.26164; start of the rise phase; end of the rise phase. The $1 \sigma$ error for the displayed data points is within the $4-6 \%$ range.

of ongoing research. There have been several attempts to explain the mechanism(s) responsible for this observational feature. Hawley \& Fisher (1992) present several models to investigate whether X-ray heating from the corona can explain the chromospheric emission observed in the optical region. Houdebine (2003) model the behavior of the Balmer and Ca II lines, applying a natural radiative cooling of the flare plasma mechanism. In this scenario the rise and decay times of the flare depend on the density of the plasma, which predicts a faster evolution of the flare in the high-temperature lines - an effect observed during several stellar flares and confirmed by us as well (see the discussion in the following paragraph).

Since the end of the flare is beyond the end of our observing run, we can only determine a lower limit of $\sim 145 \mathrm{~min}$ for the duration of the entire event. However, the major fraction of the flare is certainly covered so that the decay timescales in the individual lines can be accurately estimated. From our exponential fits, we find that the decay times decrease from $\mathrm{H} \alpha$, over $\mathrm{H} \beta$, to $\mathrm{H} \gamma$. In our sample, the latter shows the shortest decay time of $56.5 \mathrm{~min}$ while the $\mathrm{H} \delta$ and $\mathrm{H} \epsilon$ lines show longer decay time of about $70 \mathrm{~min}$. We argue that the unusually short decay time of $\mathrm{H} \gamma$ is likely spurious, potentially attributable to the unusual behavior of its light curve during the last quarter of the observation, where it seems to settle near the quiescence level. The e-folding times of the $\mathrm{Ca}$ II light curves are comparable to that of the $\mathrm{H} \alpha$ line and longer than that of the other Balmer lines.
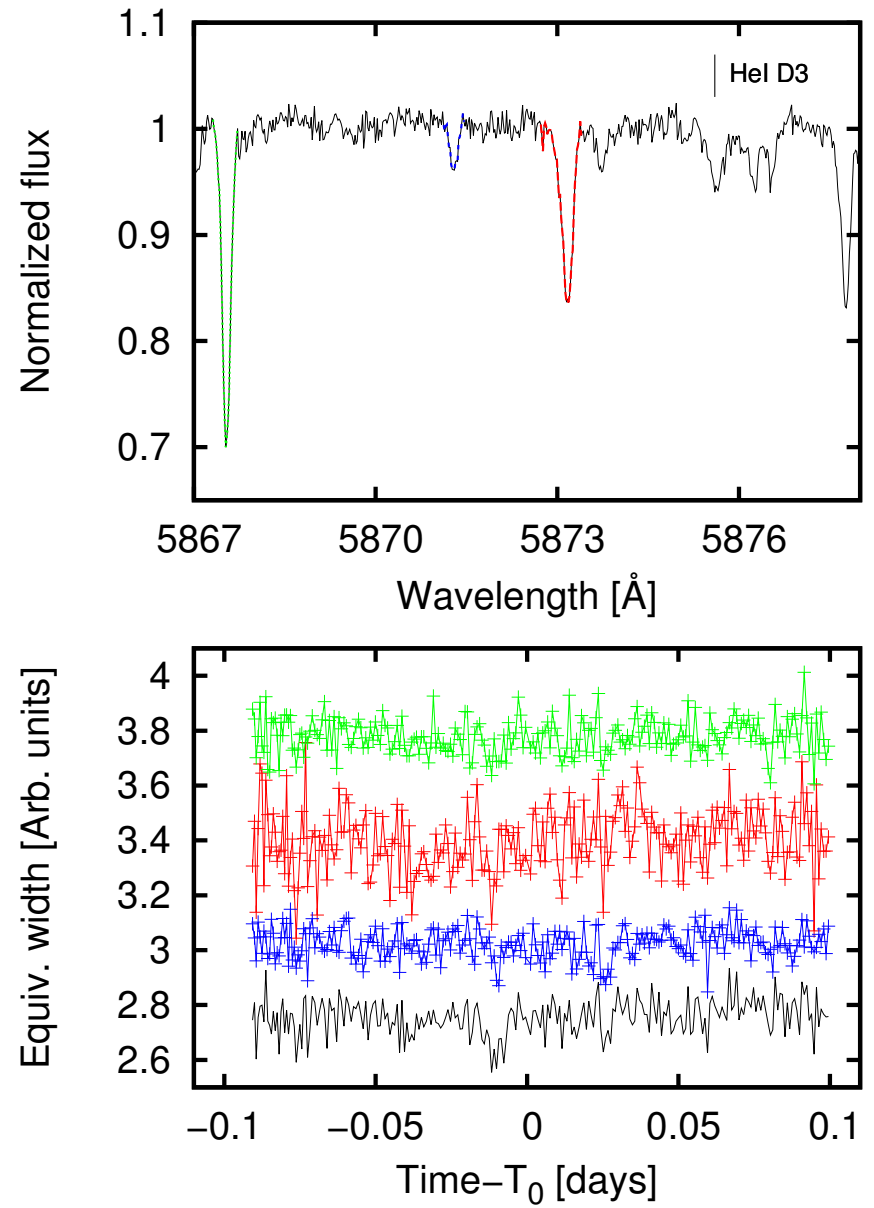

Fig. 9. Top: spectral region around He I D3 line. Neighboring photospheric lines used for the behavior check are marked with different colors. Bottom: evolution of the equivalent width of the selected photospheric lines (marked with the same colors) and of a continuum in the vicinity of the He I D3 line (black line). The curves were scaled for better comparison. The center of the $x$ axis (time $=0$ days) corresponds to the middle of the transit $T_{0}$ [HJD, UTC] $=2456109.26164$.

It is, however, not well described by an exponential after the rise phase, where it shows a plateau and evolves more gradually. The $\mathrm{Ca}$ II $\mathrm{H} \& \mathrm{~K}$ line fluxes reach smaller peak fluxes than the $\mathrm{H} \alpha$ line, but decay more slowly so that $\mathrm{H} \alpha$ and $\mathrm{Ca}$ II $\mathrm{K}$ reach about the same level of excess emission at the end of our observation.

In their analysis of a large flare on the active M2 dwarf AD Leo, Hawley \& Pettersen (1991) also find a sharper peak and faster decay in the $\mathrm{H} \gamma$ and $\mathrm{H} \delta$ lines compared to the Ca II K light curve, which shows a "rounder" evolution after the impulsive phase (see their Fig. 7). In the flare studied by Hawley \& Pettersen (1991), the peak fluxes reached by the $\mathrm{H} \gamma$ and $\mathrm{H} \delta$ lines are about three times larger than the peak flux reached by $\mathrm{Ca}$ II $\mathrm{K}$. In our case, the peak flux of the strongest Balmer line $(\mathrm{H} \alpha)$, is only about $40 \%$ higher than that observed in Ca II K, indicating a somewhat different distribution of energy among the chromospheric lines.

During the decay phase of the primary flare, we detect a smaller secondary and a tertiary flare showing decay times on the order of $10 \mathrm{~min}$, which contribute a few percent of the radiated energy in the Ca II and Balmer lines, where they could be detected. Based on our data, it remains impossible to prove a physical relation between these smaller flares and the primary event. 
Yet, no comparable flare event was observed in the first half of the observation, while we detect three flares in the second half. The existence of these secondary events is in agreement with the prediction of the standard flare model (e.g., Kopp \& Pneuman 1976). Major flares can trigger secondary flares in neighboring magnetic loop systems and also in remote active regions, which may be magnetically connected (so-called sympathetic flares, e.g., Moon et al. 2002; Wang et al. 2007).

\subsection{The behavior of $\mathrm{He}$ I D3}

The He I D3 line(s) are formed by transitions between triplet states more than $20 \mathrm{eV}$ above the ground state, which are not easily populated at photospheric and chromospheric temperatures. Therefore, the He I D3 line is weak or absent in the spectra of inactive stars, and its presence in the spectra of cool stars points to the existence of non-radiative heating processes (Saar et al. 1997). Two main mechanisms are discussed to populate the triplet states, viz., photoionization by coronal X-ray and extreme ultraviolet radiation followed by radiative recombination and collisional excitation in the hot upper chromosphere or transition region ( 20000 K, e.g., Athay \& Johnson 1960; Zirin 1975; Milkey et al. 1973); see also Garcia-Lopez et al. (1993) for a discussion, however, the simulations by Lanzafame \& Byrne (1995) indicate a minor role of coronal irradiation in the formation of the He triplet lines.

Indeed, higher activity levels can lead to stronger absorption in the He I D3 lines by more densely populating the helium triplet states. Saar et al. (1997) argue that the behavior of the line is similar to the behavior of the $\mathrm{H} \alpha$ line in cooler M dwarfs. As a consequence of rising chromospheric activity levels in very cool stars, the $n=2$ energy level becomes more populated and the equivalent width of $\mathrm{H} \alpha$ absorption increases. At the point where the level populations become collisionally controlled, the source function is determined by the temperature and as a result the line will turn into emission (e.g., Robinson et al. 1990; Cram \& Giampapa 1987, and references therein).

According to Saar et al. (1997), the behavior of the He I D3 line in active $\mathrm{G}$ and $\mathrm{K}$ dwarfs correlates with other activity indicators, such that, for instance, higher levels of $\mathrm{Ca}$ II $\mathrm{H} \& \mathrm{~K}$ line emission correspond to stronger He I D3 absorption. The same trend is observed for HD 189733 by Boisse et al. (2009), who study the evolution of $\mathrm{Ca}$ II $\mathrm{H} \& \mathrm{~K}, \mathrm{H} \alpha$, and He I D3 indices during quiescence. We find that our results are consistent with the relations between helium flux, $F_{\mathrm{D} 3}$, and stellar color, rotation period, and $\mathrm{Ca}$ II $\mathrm{H} \& \mathrm{~K}$ flux determined in the sample analysis by Saar et al. (19972; see their Figs. 3, 4, and 6). Our value of $F_{\mathrm{D} 3}=16,000 \mathrm{erg} \mathrm{cm}^{-2} \mathrm{~s}^{-1}$ agrees well with the range of $F_{\mathrm{D} 3}$ values determined by Saar et al. (1997) for K1-2V type stars (cf., their Table 1).

On the Sun, the He I D3 line is seen in emission only in more intense flares and in absorption in eruptive prominences and weaker flares (Zirin 1988, Sect. 11.3). Early solar studies have shown that the peak instants of flares observed in He I D3 can occur either at the same time as that of the Balmer lines or be delayed by up to $20 \mathrm{~min}$ (e.g. Bray 1964, and references therein). García-Alvarez et al. (2005) study the evolution of the He I D3 line during a medium, GOES C7.5 class solar flare and find that the He I D3 line remains in absorption for the whole event, and its strength is correlated with that of the $\mathrm{H} \alpha$ and

\footnotetext{
2 From the conversion between EW and helium flux it appears to us that the fluxes given by Saar et al. (1997) refer to astrophysical fluxes, which differ from physical fluxes by a factor of $\pi^{-1}$.
}

Ca II K lines. Similarly, Johns-Krull et al. (1997) report on a GOES M7.7 class solar flare also finding the He I D3 line to be in absorption throughout the entire event and absent during quiescence. However, the authors detect a constant level of absorption in He I D3 for about 40 min after the flare peak observed, for example, in $\mathrm{H} \alpha$ and soft X-rays. Only after that the He I D3 line EW starts to drop and follows the decay of the other chromospheric activity indicators.

During flares on other stars the He I D3 line has been observed in emission. For instance, Crespo-Chacón et al. (2006) detect the line studying flares on the active M-type star AD Leo and Montes et al. (1999) report He I D3 emission during a flare on the K2 dwarf LQ Hya. Our observations indicate a potential small increase in the He I D3 flux during the rise phase of the flare, which would be consistent with observations of strong flares on the Sun, thereafter a phase of increasing absorption follows. We speculate that the latter may, indeed, be caused by the flare, which may lead to a stronger population of the excited He I levels, by providing additional energy to the stellar atmosphere both in the form of local heating and more globally through the increased level of extreme-ultraviolet and soft X-ray emission illuminating the flaring hemisphere. This hypothesis would gain strong support from a return of the He I D3 absorption levels to the pre-flare level after the event. Unfortunately, the event has not been fully covered and during our observation no reversal of the trend in the He I D3 could be observed. Therefore, any conclusion on the nature of the observed behavior of the He I D3 line remains speculative.

\subsection{Flare energy budget}

The total energy released by the flare within all the studied chromospheric lines is $E_{\text {tot }}=8.7 \times 10^{31} \mathrm{erg}$ (see Table 3$)$. The largest contribution is due to the Balmer lines, followed by the Ca II H \& K lines. During a large flare on AD Leo studied by Hawley \& Pettersen (1991) only between $10 \%$ and $40 \%$ of the energy radiated in the 1200-8000 $\AA$ band, is emitted in the form of emission lines. Therefore, we estimate that the true energy budget is probably larger by a factor of between two and ten.

A number of optical spectroscopic observations of flares on K-type stars are reported in the literature (e.g., Montes et al. 1999; López-Santiago et al. 2003; Montes et al. 2005). Montes et al. (1999) study a long-duration (2 h) flare observed in the optical and ultraviolet regime on the young, single K2 dwarf LQ Hya. The total energy released in the optical chromospheric lines studied by Montes et al. ( $\mathrm{H} \alpha, \mathrm{H} \beta$, He I D3, Mg I triplet, Na I, Fe II and He I 26678$)$ is $\geq 5.7 \times 10^{33} \mathrm{erg}$, that is, two orders of magnitude higher than the value determined for the flare on HD 189733. Accordingly, the peak $\mathrm{H} \alpha$ flux is also two orders of magnitude higher. Although "our" flare on HD 189733 is comparable to or larger than the strongest solar flares, which release up to $10^{32} \mathrm{erg}$ (Priest 2014), it certainly remains moderate in the context of flares observed in young $\mathrm{K}$ dwarfs and dMe stars, which release up to about $10^{34} \mathrm{erg}$ (Hawley \& Pettersen 1991).

For a number of flares on dMe stars, Hawley \& Pettersen (1991) find that the energy released in Ca II K line is about half of that released in the $\mathrm{H} \gamma$ line. This is not the case in the flare observed on HD 189733. In fact, we find approximately the inverse ratio with about 2.5 times the energy of the $\mathrm{H} \gamma$ line released in the Ca II K line. At this point, we cannot determine whether this difference is a particularity of this specific flare or more generally valid for flares on early K-type stars such as HD 189733. 


\subsection{Extrapolating into the $X$-ray regime}

HD 189733 is a known X-ray source with a quiescent X-ray luminosity of about $1.1 \times 10^{28} \mathrm{erg} \mathrm{s}^{-1}$ (Poppenhaeger et al. 2013), and several X-ray flares have been observed on the star (e.g., Pillitteri et al. 2014). Although no simultaneous X-ray data are available for the flare studied here, we attempt a rough estimate of the level of the soft X-ray flux during the flare using the correlations between the $\mathrm{Ca}$ II $\mathrm{H} \& \mathrm{~K}$ flux and the soft X-ray flux given by Schrijver (1983).

Combining our CaII $\mathrm{H} \& \mathrm{~K}$ measurements with Eq. (6) given by Schrijver $(1983)\left(F_{\mathrm{X}}=3.4 \times 10^{5} \Delta F_{\mathrm{H}+\mathrm{K}}^{1.67}\right)$, we estimate a value of $3.2 \times 10^{5} \mathrm{erg} \mathrm{cm}^{-2} \mathrm{~s}^{-1}$ for the quiescent $\mathrm{X}$-ray surface flux of HD 189733, which compares well with the nearly identical observed value. While we therefore find the relation to also be valid in the case of HD 189733, we consider the excellent numerical correspondence a coincidence.

During the flare, the emission in the Ca II $\mathrm{H} \& \mathrm{~K}$ lines increases by about $6 \%$. Assuming that the Schrijver relation, $F_{\mathrm{X}} \sim$ $\Delta F_{\mathrm{H}+\mathrm{K}}^{1.67}$, can also be applied during the flare, we estimated a peak soft X-ray luminosity of $10^{27} \mathrm{erg} \mathrm{s}^{-1}$ for the flare. Further assuming the same e-folding time of $120 \mathrm{~min}$ as in the $\mathrm{Ca}$ II $\mathrm{H} \& \mathrm{~K}$ lines also in X-rays, we estimated that a total energy of $7 \times 10^{30}$ erg was released by the flare in soft X-rays. Alternatively, we could apply the relation, $L_{\mathrm{X}}=31.6 \times L(\mathrm{H} \gamma)$, derived from flare observations on nearby dMe stars (Butler et al. 1988) and arrive at an estimate of $14 \times 10^{30} \mathrm{erg}$ for the total energy radiated at soft X-rays, which agrees with the former estimate to within a factor of two. According to these estimates, the X-ray flares reported on by Pillitteri et al. (2014) are about an order of magnitude stronger with mean flare luminosities of several times $10^{27} \mathrm{erg} \mathrm{s}^{-1}$ and total energies of several times $10^{31} \mathrm{erg}$ released in X-rays.

As the planet and the flare were located on the same stellar hemisphere, the planetary atmosphere was clearly exposed to the full flare radiation. However, we consider a transient rise in $\mathrm{X}$-ray emission by $\sim 10 \%$ too small to significantly affect the hydrodynamic escape of the planet's atmosphere, which can only use about $1 \%$ of the irradiated energy to drive atmospheric escape (Salz et al. 2016). Yet, significantly stronger flares could, indeed, lead to a significant change in the atmospheric structure (Lecavelier des Etangs et al. 2012) and the impact of many smaller flares may also accumulate.

\subsection{Star-planet interaction}

As discussed in Sect. 1, the activity and proximity of HD 189733 and its planet make this system an ideal target for studies of star-planet interaction (SPI). SPI was theoretically predicted by Cuntz et al. (2000) and was later claimed by, for example, Shkolnik et al. $(2005,2008)$ as a modulation of selected lines in the spectra of stars with close-in planets phased with the planetary orbit. SPI is still a controversial subject and a topic of much ongoing research.

Fares et al. (2010) use spectropolarimetric measurements of this system covering two stellar rotation cycles and find that the residuals of the $\mathrm{H} \alpha$ and $\mathrm{Ca}$ II $\mathrm{H} \& \mathrm{~K}$ lines with respect to an average line profile are periodic and modulated with the rotation period of the star, rather the orbital period of the planet; hence Fares et al. (2010) do not find any evidence for SPI. Cohen et al. (2011) perform time-dependent MHD modeling of SPI in the HD 189733 system and show that in their simulations reconnection events can occur during a specific orbital phase. Such events could then be responsible for the mass loss from the planetary magnetosphere, causing a hotspot on the surface of the star observed as modulation in Ca II H \& $\mathrm{K}$ lines. As described in Sect. 1, Pillitteri et al. (2010, 2011, 2014) observe flares occurring in HD 189733 in quite a tight orbital phase interval, possibly caused by the orbiting exoplanet. Their findings are supported by the models of Lanza (2012), who predict a modulation of coronal flaring activity with the orbital phase of the planet.

In our case, we observe the flare starting in the middle of the primary transit. To our knowledge, so far there has been no observation of increased stellar activity during a primary transit. There are more than 20 observations of HD 189733 during a primary transit (e.g., Redfield et al. 2008; Wyttenbach et al. 2015; Cauley et al. 2017). Although flares were not specifically studied by these authors, it appears to be very plausible that an event of a similar magnitude to that observed by us would have been noticed by the authors. Thus the hypothesis that this may be a recurrent event seems unlikely. To prove this correlation, we would need more observation of a flare during a transit. Nevertheless, with respect to the simulations of Cohen et al. (2011), it could have happened that the planet around HD 189733 has encountered a locally enhanced magnetic field of a host star and induced a flare. So clearly more observations of activity related features are required to arrive at firm conclusions with respect to to the presence of SPI effects in HD 189733.

\section{Summary and conclusion}

Using spectra obtained with the UVES spectrograph we have presented a temporally resolved spectral analysis of the evolution in the chromospheric lines $\mathrm{H} \alpha, \mathrm{H} \beta, \mathrm{H} \gamma, \mathrm{H} \delta, \mathrm{H} \epsilon, \mathrm{He}$ I D3, Ca II $\mathrm{H} \& \mathrm{~K}$, and Ca II IR triplet during a flare observed on the planet host star HD 189733; the flare started shortly after mid-transit at $\sim$ MJD 56109.26 , and finished after our observation. To study the Ca II H \& $\mathrm{K}$ lines, we first used the chromospheric $\log R_{\mathrm{HK}}^{\prime}$ activity index (Fig. 3), subsequently, we studied the excess emission in the cores of all the Balmer lines, the $\mathrm{Ca}$ II H \& K, and Ca II IRT lines, and the He I D3 line relying on the flux calibration of our spectra (Fig. 7). The energetics and characteristics of the flare are summarized in Tables 2-5.

The Balmer and $\mathrm{Ca}$ II lines show a typical flare signature with a fast impulsive phase with a duration of $\sim 28 \mathrm{~min}$ and a slower decay phase, which lasts for at least two hours. Nonetheless, there is a clear difference in the temporal evolution of the Balmer and $\mathrm{Ca}$ II lines with the former showing a distinct peak followed by a quasi-exponential decay and the latter a more gradual evolution after the impulsive phase. Qualitatively, this evolution is consistent with previous flare observations in dMe stars.

The evolution of the He I D3 line follows a different temporal pattern than the other chromospheric lines. It shows a potential small emission episode during the impulsive phase of the flare and afterwards, with a substantial delay, turns into absorption compared to the pre-flare level. While this behavior may be related to the flare, a unique association is difficult to establish based on the available data.

According to our analysis, the total energy released in the studied optical chromospheric lines (except for the He I D3 line) is $\sim 8.7 \times 10^{31} \mathrm{erg}$, which puts the observed flare between the most energetic solar flares and moderate stellar flares observed on K-type and M-type stars. We observed a smaller secondary and a tertiary flare occurring during the decay phase of the primary event. Although it cannot be proved, they may well be triggered by the primary flare. As our observations remain so far the only in-transit flare observations in HD 189733, although many observations during a transit have already been carried out (e.g., 
Redfield et al. 2008; Wyttenbach et al. 2015; Triaud et al. 2009), we consider a SPI scenario as proposed by Pillitteri et al. (2014) to be unlikely in this case and we rather consider the flare timing a chance coincidence.

The cores of selected optical spectral lines are used for the exoplanet atmosphere detection (see e.g., Charbonneau et al. 2002; Redfield et al. 2008, and references therein). Our analysis shows that the activity of an exoplanet host star can affect cores of a wide range of optical lines and must therefore be taken into account when studying exoplanetary atmospheres, as demonstrated by Khalafinejad et al. (2017).

Acknowledgements. The authors would like to thank Prof. Petr Heinzel for helpful comments on the manuscript. This work has been supported by the DFG grants CZ 222/1-1 and SCHM 1032/37-1. T.K. and S.K. acknowledge support from the RTG 1351 "Extrasolar planets and their host stars".

\section{References}

Agol, E., Cowan, N. B., Knutson, H. A., et al. 2010, ApJ, 721, 1861 Andretta, V., Giampapa, M. S., \& Jones, H. P. 1995, Ir. Astron. J., 22 Athay, R. G., \& Johnson, H. R. 1960, ApJ, 131, 413

Bakos, G. Á., Pál, A., Latham, D. W., Noyes, R. W., \& Stefanik, R. P. 2006, ApJ, 641, L57

Baliunas, S. L., Donahue, R. A., Soon, W. H., et al. 1995, ApJ, 438, 269

Benz, A. O. 2008, Liv. Rev. Sol. Phys., 5, 1

Boisse, I., Moutou, C., Vidal-Madjar, A., et al. 2009, A\&A, 495, 959

Bouchy, F., Udry, S., Mayor, M., et al. 2005, A\&A, 444, L15

Bray, R. J. 1964, Z. Astrophys., 60, 207

Butler, C. J., Rodono, M., \& Foing, B. H. 1988, A\&A, 206, L1

Canfield, R. C., Gunkler, T. A., \& Ricchiazzi, P. J. 1984, ApJ, 282, 296

Castelli, F., \& Kurucz, R. L. 2004, ArXiv Astrophysics e-prints [arXiv:astro-ph/0405087]

Cauley, P. W., Redfield, S., \& Jensen, A. G. 2017, AJ, 153, 217

Charbonneau, D., Brown, T. M., Noyes, R. W., \& Gilliland, R. L. 2002, ApJ, 568,377

Chmielewski, Y. 2000, A\&A, 353, 666

Cohen, O., Kashyap, V. L., Drake, J. J., et al. 2011, ApJ, 733, 67

Cram, L. E., \& Giampapa, M. S. 1987, ApJ, 323, 316

Crespo-Chacón, I., Montes, D., García-Alvarez, D., et al. 2006, A\&A, 452, 987

Cuntz, M., Saar, S. H., \& Musielak, Z. E. 2000, ApJ, 533, L151

Cutispoto, G., Pastori, L., Guerrero, A., et al. 2000, A\&A, 364, 205

Czesla, S., Klocová, T., Khalafinejad, S., Wolter, U., \& Schmitt, J. H. M. M. 2015, A\&A, 582, A51

Fares, R., Donati, J.-F., Moutou, C., et al. 2010, MNRAS, 406, 409

Feldman, U., Liggett, M., \& Zirin, H. 1983, ApJ, 271, 832

Filliben, J. J., \& McKinney, J. E. 1972, J. Res. Natl. Stand. Sec. B., 76B, 179

Fuhrmeister, B., Liefke, C., Schmitt, J. H. M. M., \& Reiners, A. 2008, A\&A, 487, 293

García-Alvarez, D., Jevremović, D., Doyle, J. G., \& Butler, C. J. 2002, A\&A, 383,548

García-Alvarez, D., Johns-Krull, C. M., Doyle, J. G., et al. 2005, A\&A, 444, 593

Garcia-Lopez, R. J., Rebolo, R., Beckman, J. E., \& McKeith, C. D. 1993, A\&A, 273,482

Gray, R. O., \& Corbally, C. J. 1994, AJ, 107, 742

Gray, R. O., Corbally, C. J., Garrison, R. F., McFadden, M. T., \& Robinson, P. E. 2003, AJ, 126, 2048

Hawley, S. L., \& Fisher, G. H. 1992, ApJS, 78, 565

Hawley, S. L., \& Pettersen, B. R. 1991, ApJ, 378, 725

Henry, G. W., \& Winn, J. N. 2008, AJ, 135, 68

Henry, T. J., Soderblom, D. R., Donahue, R. A., \& Baliunas, S. L. 1996, AJ, 111 439

Houdebine, E. R. 2003, A\&A, 397, 1019

Johns-Krull, C. M., Hawley, S. L., Basri, G., \& Valenti, J. A. 1997, ApJS, 112, 221
Kausch, W., Noll, S., Smette, A., et al. 2015, A\&A, 576, A78

Khalafinejad, S., von Essen, C., Hoeijmakers, H. J., et al. 2017, A\&A, 598, A131

Knutson, H. A., Howard, A. W., \& Isaacson, H. 2010, ApJ, 720, 1569

Koen, C., Kilkenny, D., van Wyk, F., \& Marang, F. 2010, MNRAS, 403, 1949

Kopp, R. A., \& Pneuman, G. W. 1976, Sol. Phys., 50, 85

Kowalski, A. F., Cauzzi, G., \& Fletcher, L. 2015, ApJ, 798, 107

Kurucz, R. L. 1970, SAO Spec. Rep., 309

Lalitha, S., Fuhrmeister, B., Wolter, U., et al. 2013, A\&A, 560, A69

Landman, D. A. 1981, ApJ, 244, 345

Lang, K. R. 2009, The Sun From Space (Springer-Verlag)

Lanza, A. F. 2012, A\&A, 544, A23

Lanzafame, A. C., \& Byrne, P. B. 1995, A\&A, 303, 155

Lecavelier des Etangs, A., Bourrier, V., Wheatley, P. J., et al. 2012, A\&A, 543, L4

Leka, K. D., Canfield, R. C., McClymont, A. N., et al. 1993, ApJ, 411, 370

Linsky, J. L., Hunten, D. M., Sowell, R., Glackin, D. L., \& Kelch, W. L. 1979, ApJS, 41, 481

López-Santiago, J., Montes, D., Fernández-Figueroa, M. J., \& Ramsey, L. W. 2003, A\&A, 411, 489

Madhusudhan, N., Knutson, H., Fortney, J. J., \& Barman, T. 2014, Protostars and Planets VI, 739

Melo, C., Santos, N. C., Pont, F., et al. 2006, A\&A, 460, 251

Middelkoop, F. 1982, A\&A, 107, 31

Milkey, R. W., Heasley, J. N., \& Beebe, H. A. 1973, ApJ, 186, 1043

Montes, D., Saar, S. H., Collier Cameron, A., \& Unruh, Y. C. 1999, MNRAS, 305,45

Montes, D., López-Santiago, J., Crespo-Chacón, I., \& Fernández-Figueroa, M. J. 2005, in 13th Cambridge Workshop on Cool Stars, Stellar Systems and the Sun, eds. F. Favata, G. A. J. Hussain, \& B. Battrick, ESA SP, 560, 825

Moon, Y.-J., Choe, G. S., Park, Y. D., et al. 2002, ApJ, 574, 434

Notsu, S., Honda, S., Notsu, Y., et al. 2013, PASJ, 65, 112

Noyes, R. W., Hartmann, L. W., Baliunas, S. L., Duncan, D. K., \& Vaughan, A. H. 1984, ApJ, 279, 763

Pillitteri, I., Wolk, S. J., Cohen, O., et al. 2010, ApJ, 722, 1216

Pillitteri, I., Günther, H. M., Wolk, S. J., Kashyap, V. L., \& Cohen, O. 2011, ApJ, 741, L18

Pillitteri, I., Wolk, S. J., Lopez-Santiago, J., et al. 2014, ApJ, 785, 145

Pillitteri, I., Maggio, A., Micela, G., et al. 2015, ApJ, 805, 52

Pont, F., Gilliland, R. L., Moutou, C., et al. 2007, A\&A, 476, 1347

Poppenhaeger, K., Schmitt, J. H. M. M., \& Wolk, S. J. 2013, ApJ, 773, 62

Priest, E. 2014, Magnetohydrodynamics of the Sun (Cambridge: Cambridge University Press)

Redfield, S., Endl, M., Cochran, W. D., \& Koesterke, L. 2008, ApJ, 673, L87

Robinson, R. D., Cram, L. E., \& Giampapa, M. S. 1990, ApJS, 74, 891

Saar, S. H., Huovelin, J., Osten, R. A., \& Shcherbakov, A. G. 1997, A\&A, 326 , 741

Salz, M., Schneider, P. C., Czesla, S., \& Schmitt, J. H. M. M. 2016, A\&A, 585, L2

Schmitt, J. H. M. M. 1997, A\&A, 318, 215

Schmitt, J. H. M. M., Fleming, T. A., \& Giampapa, M. S. 1995, ApJ, 450, 392

Schrijver, C. J. 1983, A\&A, 127, 289

Shkolnik, E., Walker, G. A. H., Bohlender, D. A., Gu, P.-G., \& Kürster, M. 2005, ApJ, 622, 1075

Shkolnik, E., Bohlender, D. A., Walker, G. A. H., \& Collier Cameron, A. 2008, ApJ, 676, 628

Smette, A., Sana, H., Noll, S., et al. 2015, A\&A, 576, A77

Torres, G., Winn, J. N., \& Holman, M. J. 2008, ApJ, 677, 1324

Triaud, A. H. M. J., Queloz, D., Bouchy, F., et al. 2009, A\&A, 506, 377

van Leeuwen, F. 2007, A\&A, 474, 653

Vernazza, J. E., Avrett, E. H., \& Loeser, R. 1981, ApJS, 45, 635

Wang, H., Liu, C., Jing, J., \& Yurchyshyn, V. 2007, ApJ, 671, 973

Wilson, O. C. 1968, ApJ, 153, 221

Wright, J. T., Marcy, G. W., Butler, R. P., \& Vogt, S. S. 2004, ApJS, 152, 261

Wyttenbach, A., Ehrenreich, D., Lovis, C., Udry, S., \& Pepe, F. 2015, A\&A, 577, A62

Zirin, H. 1975, ApJ, 199, L63

Zirin, H. 1988, Astrophysics of the Sun (Cambridge and New York: Cambridge University Press) 